\title{
Diversity of macro fungus across three altitudinal ranges in Lore Lindu National Park, Central Sulawesi, Indonesia and their utilization by local residents
}

\author{
Y. YUSRAN ${ }^{1, \boldsymbol{v}}$, E. ERNIWATI ${ }^{1}$, D. WAHYUNI ${ }^{1}$, R. RAMADHANIL ${ }^{2}$, A. KHUMAIDI ${ }^{3}$ \\ ${ }^{1}$ Department of Forestry, Faculty of Forestry, Universitas Tadulako. Jl. Soekarno-Hatta Km. 9, Palu 94118, Central Sulawesi, Indonesia. \\ Tel./fax.: +62-451-422611, ’email: yyusranyusran610@gmail.com \\ ${ }^{2}$ Department of Biology, Faculty of Mathematics and Natural Science, Universitas Tadulako. Jl. Soekarno-Hatta Km. 9, Palu 94118, Central Sulawesi, \\ Indonesia \\ ${ }^{3}$ Department of Pharmacy, Faculty of Mathematics and Natural Science, Universitas Tadulako. Jl. Soekarno-Hatta Km. 9, Palu 94118, Central Sulawesi, \\ Indonesia
}

Manuscript received: 6 October 2020. Revision accepted: 20 December 2020.

\begin{abstract}
Yusran Y, Erniwati E, Wahyuni D, Ramadhanil R, Khumaidi A. 2021. Diversity of macro fungus across three altitudinal ranges in Lore Lindu National Park, Central Sulawesi, Indonesia and their utilization by local residents. Biodiversitas 22: 199-210. A large amount of biodiversity research has been carried out in Lore Lindu National Park, a major biodiversity center in Central Sulawesi, Indonesia, but none have investigated the biodiversity of macrofungi and their traditional utilization. Therefore, this study aimed to explore the diversity of macro fungus in Lore Lindu National Park, and to identify their potential uses as food sources and medicinal uses by the local residents living around Lore Lindu National Park. Exploration of macrofungus species in Lore Lindu National Park was done at three locations representing three altitudinal ranges: (i) $<500 \mathrm{~m}$ above sea level (asl); (ii) $500-1500 \mathrm{~m}$ asl; and $>1500 \mathrm{~m}$ asl. Ten plots were placed in two major lines with a $100 \mathrm{~m}$ distance between plots in each sampling location. All macrofungi within the observation plots were then documented and identified. Ethnomycological studies were done by asking questionnaire to selective respondents, group discussion, and pictorial presentation studies to random respondents in five villages located in the buffer zone of the national park area. This study found 172 species (including unidentified species/sp.) from 33 families of macro fungus in Lore Lindu National Park in which 159 of them belong to the Basidiomycota division, while 13 of them were of the Ascomycota division. Our results also showed varying diversity of macrofungus at different altitudes. At the elevation of $<500 \mathrm{~m}$ asl, as many 77 species were found, while 117 and 142 species were found at the elevation of 500-1500 and $>1500 \mathrm{~m}$ asl, respectively. Marasmius spp and Hygrocybe spp were the most abundant genera, and nine species (i.e. Schizophyllum commune, Termytomyces sp, Auricularia auricularjudge, Auricularia sp., Pleurotus ostreatus, Ganoderma lucidum, Xylaria sp., Agaricus sp. dan Lentinus sajor-caju) were utilized as a food source and in traditional medicine by the residents around the national park area.
\end{abstract}

Keywords: Ethnomycology, Lore Lindu National Park, macro-fungi diversity, traditional knowledge

\section{INTRODUCTION}

Indonesia is a tropical country with rich natural resources, including high diversity of flora, fauna and microorganisms. Sulawesi is an island in Indonesia that biogeographically is located within the Wallace lines, a region between Asia and Australasia lines (Wallace 1869) and between the Laurasian and Gondwanan plates (Corlett and Primack 2006). This unique biogeographical position along with suitable environmental conditions make Sulawesi have enormous abundance of species diversity, including numerous endemic species. About $15 \%$ of flowering plant species from Sulawesi have been identified and categorized as endemic species (Whitten et al. 1987). Van Balgooy et al. (Van Balgooy et al. 1996) recognized that 933 new plant species originated from Sulawesi, and found that 112 species were endemic. A plant group with high diversity and endemism in Sulawesi is fungi.

Fungi is categorized as the second-largest biotic community after insects (Sarbhoy et al. 1996). Hawksworth \& Lücking (2017) estimated that fungi emerged around
2,2-3,8 million species in the world. Macrofungus is a major component of the fungal kingdom. This group of fungi plays important roles in the wider natural ecosystem, including as decomposers and food sources for animals (Tan et al. 2015). Ecologically, macrofungus can be classified into three major groups: saprophytes, parasites, and symbiotic species. Almost all terrestrial macrofungus are saprophytes or mycorrhizal symbionts, but some of them are plant pathogens. Taxonomically, most of them belong to Basidiomycota or Ascomycota, with a few being a member of Zygomycota (Mueller et al. 2007). Based on habitat characteristics, the known macrofungus are classified into three, whether they are terricolous saprophytic, wood-inhabiting, or ectomycorrhizal (Kutszegi et al. 2015).

Some wild macrofungus species are edible and considered as important food sources of communities in many countries due to their nutrient contents. An estimated 1069 species of macrofungus have been used as sources of food worldwide (Boa 2004). In some Asian countries, macrofungus is also traditionally used for medicinal 
purposes (Manzi et al. 1999). Ethnomycological research was done by Rosemary-Kinge et al. (2017) and Nema et al. (2014) revealed the uses of macrofungus from a variety of perspectives, such as for consumption, nutrients, and medicines. This research studied the potential utilizations of macrofungus in agro-industry sector and its commercial benefits in medical applications in developed countries. In line with these studies, Okhuoya et al. (2010), Herawati et al. (2016), and Hussein et al. (2016) summarized that macrofungus need to be successfully domesticated to unlock their commercialization potential. Once domesticated, they can become economically important commodities as food sources, medicines, biological control agents, and raw sources of chemical substances in pharmaceutical industry (Duarte et al. 2006). Kim \& Song (2014) stated that many macrofungus show important commercial characteristics, such as high protein, fiber, vitamin, and mineral contents, with low-fat levels, and produce medical compounds known to treat cancer and diabetes. For example, the macro fungus Trametes versicolor is known as decomposer of resistant organic pollutants (Tran et al. 2010; Tran et al. 2013), and is used as bioremediation agent for industrial waste and heavy metals (Demirbas 2000; Kalac et al. 2004). It has also been reported that macrofungus in the ectomycorrhizal group is one of the most important non-wood forest products in international trade, especially from the genera of Amanita, Boletus, Tuber, Tricholoma, Cantharellus, and Lactarius, along with and saprophytic fungi from the genera of Morchella, Pleurotus, and Agaricus.

While Indonesia ranks second in term biodiversity richness after Brazil (Muchlisin and Azizah 2009), yet information on the diversity of macrofungus and their uses local communities are scarce. There are still abundant tropical fungal species that are yet to be identified, described, and documented. This lack of work is primarily caused by minimal research effort and the limited number of fungal taxonomists in Indonesia that can carry out rigorous identification, especially of medicinal and edible fungi. Recently, research done by several groups worldwide were not able to provide sufficient data on the biodiversity of fungi and their applications (Chang and lee 2004; Retnowati 2007; Haryati and Azrianingsih 2012; Enas et al. 2016; Herawati et al. 2016; Arko et al. 2017; Marzuki et al. 2017; Foo et al. 2018; Kang et al. 2013; Khastini et al. 2018).

The lack of biodiversity data on macrofungus is also a specific problem for Sulawesi, especially in Central Sulawesi Province. Although this province has a vast-range of forests in comparison to the other five provinces, no work has been done on macrofungus diversity or their ethnomycological uses. This work aimed to explore fungal diversity in Lore Lindu National Park, a major biodiversity center in Central Sulawesi, and to identify their potential uses as food sources and natural bioactive products. This aim was achieved by carried out sampling and species identification of macrofungus in the Lore Lindu National
Park area, and complemented with surveying residents on their level of knowledge and uses of macrofungus.

\section{MATERIALS AND METHODS}

\section{Study period and area}

This study was conducted from January to August 2017 in Lore Lindu National Park. The park is located between Sigi District and Poso District, Central Sulawesi Province, Indonesia. This area lies between $119^{\circ} 90^{\prime}-120^{\circ} 16^{\prime} \mathrm{E}$ and $1^{\circ} 8^{\prime}-1^{\circ} 3^{\prime} \mathrm{S}$ with elevation from 200 to $2610 \mathrm{~m}$ above the sea level. The park has precipitation of $1200-2200 \mathrm{~mm}$ per year with average temperatures of $26^{\circ} \mathrm{C}$ (TNC/BTNLL 2002). Lore Lindu National Park has legal status as a National Park based on the Decree of the Minister of Forestry, Republic of Indonesia, No. 593/Kpts-II/93. Lore Lindu National Park has an area of $\pm 229,177.5$ ha. This area has its own uniqueness because most of its constituent components are representative of the flora and fauna of the Sulawesi mountain forests, where $90 \%$ of the total area is at an altitude above $1000 \mathrm{~m}$ above sea level (Pitopang 2006). In this area, there are also several types of ecosystems such as lowland rain forest (200-1000 m asl), montane rain forest (1000-2500 m asl), grasslands, and highland swamp forests (TNC 2001).

Exploration of macrofungus species in Lore Lindu National Park was done at three altitudinal ranges. First site had elevation range of $<500 \mathrm{~m}$ above sea level (asl), and was administratively part of Bora Village, Sigi Biromaru Sub-district, Sigi District. The other two sites had elevation range of 500-1500 $\mathrm{m}$ asl (part of the Nokilalaki Trekking line), and $>1500 \mathrm{~m}$ asl (part of Sedoa Village), both were administratively in Lore Utara Sub-district, Poso District. The sampling area was located around $\pm 30-80 \mathrm{~km}$ southwest of Palu City, the capital of Central Sulawesi Province (Figure 1).

\section{Survey, collection, and identification of macrofungus}

Sampling locations were chosen based on the accessibility of the area and macro fungus occurrence (Rosemary-Kinge et al. 2011). Observation plots were deployed at each different altitudinal range, according to Engola et al. (2007). Ten observation plots with size of $5 \times 200 \mathrm{~m}(0.1 \mathrm{Ha})$ were placed in two major lines, with 100 $\mathrm{m}$ distance between plots in primary forest in each location, resulting in 10 replicates. The observation was complete after 8 months starting from January 2017. Macro fungus observation was done by whole body collection of fungi that appeared in logs, rooted wood, leaf litter, animal waste, or soil. The detail on the morphological characteristics and the native condition of the environment were documented using a digital camera. Fruit body collections were maintained carefully to prevent sporocarp damage. Excess soil that stuck to the sporocarp was removed, the fruit body carefully dried using a tissue, and then transferred to an envelope, which was labeled to prevent spore contamination from different fungal species. 


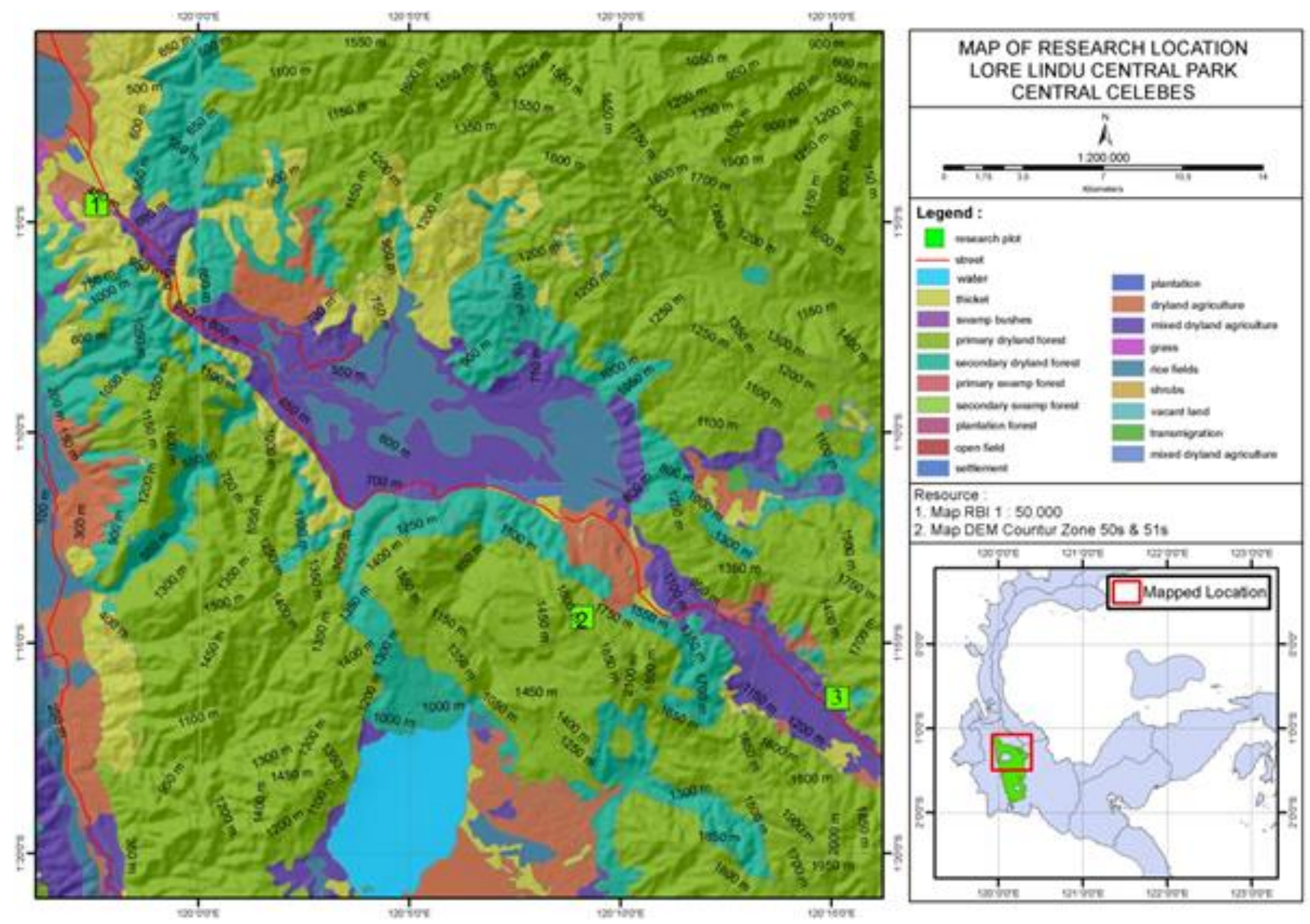

Figure 1. Map of sampling sites in Lore Lindu National Park, Central Sulawesi, Indonesia. Site 1: altitude $<500 \mathrm{~m}$ asl; Site 2: $>1500 \mathrm{~m}$ asl; and Site 3: 500-1500 m asl)

The fungi collection was then transferred to the laboratory for examination under a microscope and further identification. The sporocarp was dried using a drying machine with a maximum temperature of $35^{\circ} \mathrm{C}$ for 24 hours. Microscopy characteristics, such as form and color of the spore, were then identified. Fungal identification was carried out by macro and micro characteristics (Bates 2006; Desjardin et al. 2004; Desjardin et al. 2000; Hemmes and Desjardin 2002; Index Fungorum 2017; Mushroom Expert 2017; Stadler et al. 2014). Micro-features were observed using a standard microscope (Andrew et al. 2013). Samples were then stored in the Forest Science Laboratory, Faculty of Forestry, Universitas Tadulako, Palu, Indonesia. Data of macro fungus diversity was then analyzed using the descriptive statistics available in Microsoft Excel 2010.

\section{Ethnomycology documentation}

Interviews with residents living around Lore Lindu National Park were employed to complement the biodiversity information. Respondents of both genders were chosen in every village, mainly targeting respondents who served as village shaman (dukun kampung=Sando), traditional public figures, and fungus sellers in traditional markets that were located in the supporting zone of the national park. These respondents were targeted as they were assumed to be familiar with macrofungus that is edible and has medicinal purposes. Respondents were chosen in five main villages that were located in the supporting zone of the national park, which are Sedoa, Kamarora A, Kamarora B, Lembantongoa, and Tongoa. Residents of those villages are dominated by the Kaili Da'a tribe, with a few from the Bugis, Bada, Seko, Behoa, and Toraja tribes, except in Sedoa Village that is occupied by the Lore tribe. Fifty questionnaires were given to the respondents in each village, followed by an interview and discussion. Questions on basic data (name, age, occupation, migration history, land ownership, and family members) were asked along with questions on the macrofungus known to grow around them (local name, description, time of emergence, growth place, how to use them as medicine or food, which part of the fungus residents prefer to use relating to the disease they are trying to cure, and how to use them). Furthermore, a group discussion and pictorial presentation were also conducted by showing photos of macro fungus that had already been collected in the national park area to the respondents. Statistical analysis was done to analyze ethnomycological data obtained from the interviews. 


\section{RESULTS AND DISCUSSION}

\section{Macro fungus diversity}

This study found 172 species (including unidentified species/sp.) from 33 families of macro fungus in Lore Lindu National Park in which 159 of them belong to the Basidiomycota division, while 13 of them were of the Ascomycota division (Table 1). Around 24 species of macrofungus were identified as the members of the Polyporaceae family, 18 species the Russulaceae, 17 species the Marasmiaceae, 13 species the Hygrophoraceae, 10 species the Agaricaceae, 9 species the Auriculariaceae, 7 species the Ganodermataceae, 6 species for each family Phallaceae, Sarcoscyhaceae and Strophariaceae, 5 species the Amanitaceae, 4 species for each family Boletaceae, Mycenaceae, Pleurotaceae and Xylariaceae, 3 species for each family Entolomataceae, Geastraceae, Helotiaceae, Hymenogastraceae, Lyophyllaceae, and Scizophyllaceae, 2 species for each family Bolbitiaceae, Fumitopsidaceae, Gomphaceae and Gomphidiaceae, and 1 species of each belonged to the Clavulinaceae, Hymenochaeteceae, Inocybaceae, Niaceae, Psathyrellaceae, Stereaceae, Tricholomataceae and Tremelaceae families. Three families, i.e. Helotiaceae, Sarcoscyphaceae and Xylariaceae, belong to the Ascomycota division, while the others belong to the Basidiomycota. The family and the species of each family are presented in Figure 2.

Our results also showed varying diversity of macrofungus at different altitudes. At the elevation of $<500$ $\mathrm{m}$ asl, as many 77 species were found, while 117 and 142 species were found at the elevation of 500-1500 and >1500 $\mathrm{m}$ asl, respectively. This finding suggests that the diversity of macrofungus is positively correlated with increasing altitude (Figure 3). This result contradicts previous research, which reported the diversity of fungus decreased with increasing altitude $(30,37,48$,$) . Our observations also$ indicate that 22 species of fungi were only found at the elevation of $<500 \mathrm{~m}$ asl, four species at the elevation of $500-1500 \mathrm{~m}$ asl, and 31 species at the elevation of $>1500$ $\mathrm{m}$ asl. Further analysis revealed varying abundance across altitudes, with 48 species were found at all three altitudes, 53 species were found at both the altitudes of $<500 \mathrm{~m}$ and $500-1500 \mathrm{~m}$ asl, 50 species were found at both the elevations of $<500 \mathrm{~m}$ and $>1500 \mathrm{~m}$ asl, and 109 were species found at both the elevations of $500-500 \mathrm{~m}$ and $>1500 \mathrm{~m}$ asl (Figure 4).

The different abundances of macrofungus species found in the sampling locations could be caused by many factors, especially the altitude itself, which also relates to different plant composition in the area, then followed by the microclimate. Previous research reported that microclimatic environmental variables, such as temperature, humidity, soil humidity, rainfall intensity, organic composition, leaf litter quality, hummus, methods of collection, and the plant-soil condition shape the environment and determine the distribution and diversity of macrofungus (Baptista et al. 2010; Bergemann and Largent 2010; Conn and Dighton 2000; Engola et al. 2007; Ferris et al. 2000; Kutszegi et al. 2015; Lopez-Quintero et al. 2012; Luo et al. 2016; Packham et al. 2002; Pushpa and Purushothama 2012). These findings are also supported by Angelini et al. (2015), who suggested that macro fungus emergence is highly related to the plant type that dominates the forest.

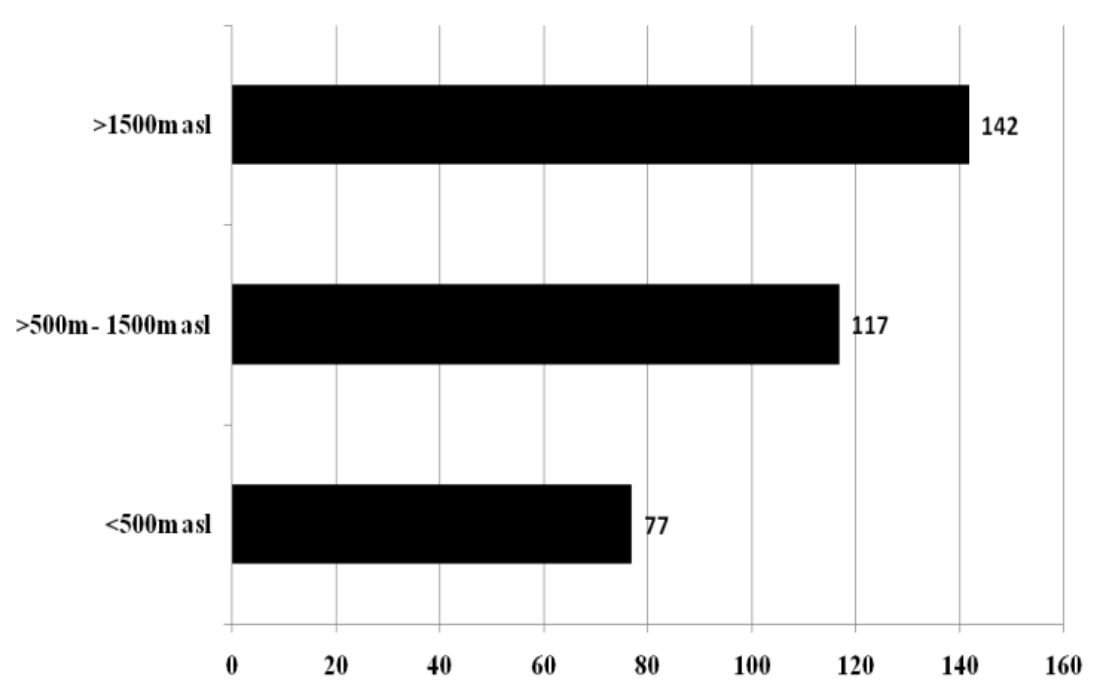

Figure 3. Number of species of macro fungus in the Lore Lindu National Park across altitudinal ranges. Note: asl: above sea level 


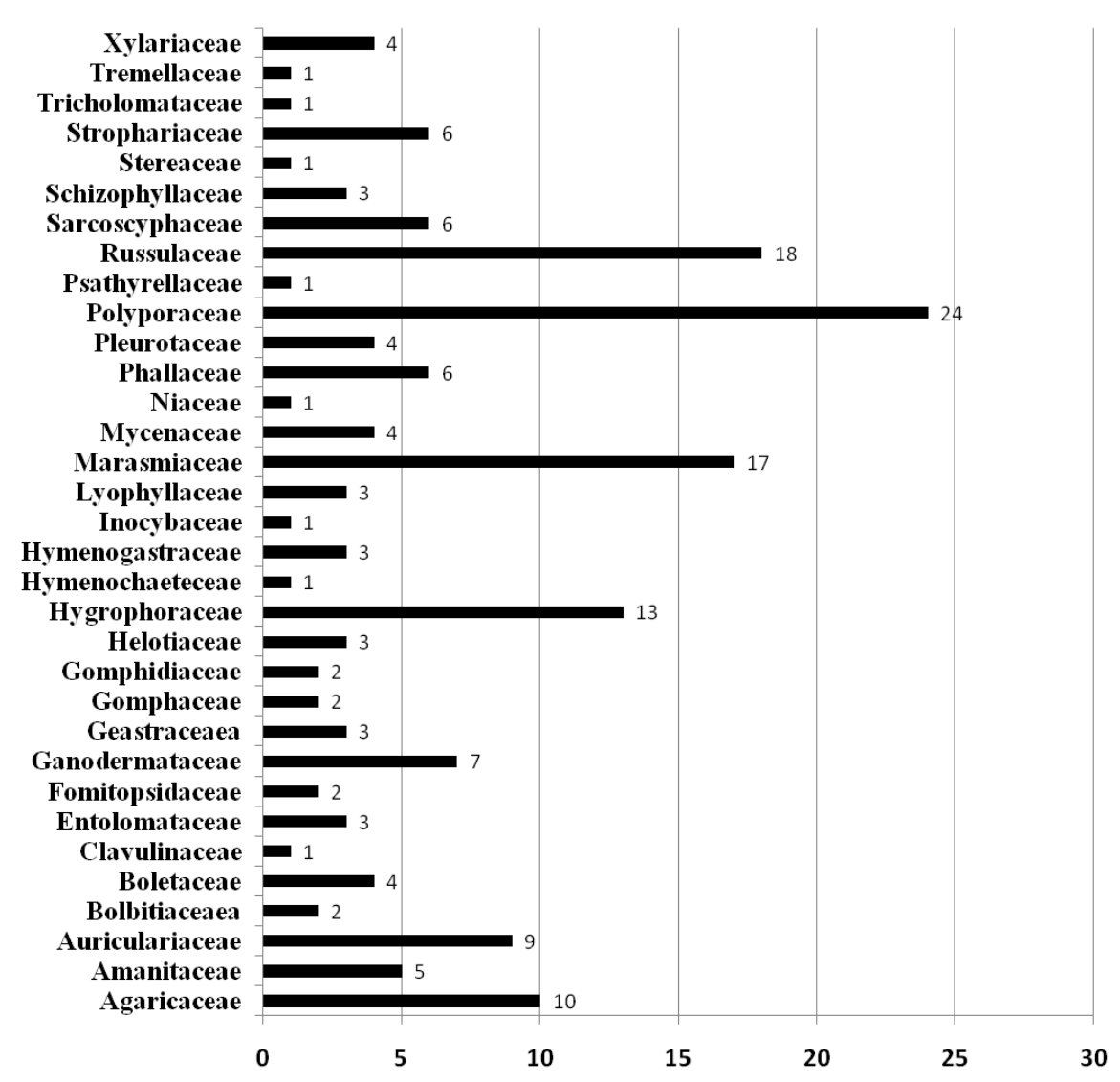

Figure 2. Family and the species of each family of macrofungus in Lore Lindu National Park, Central Sulawesi, Indonesia

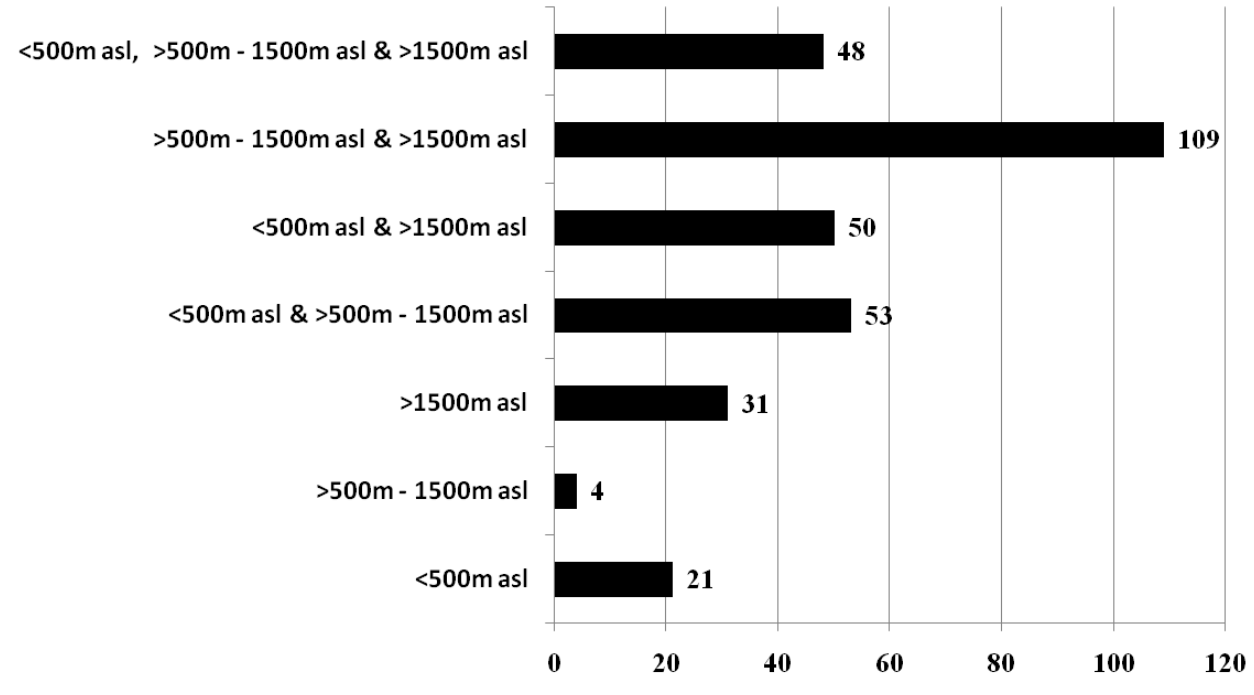

Figure 4. Number of species of macro fungus in the Lore Lindu National Park, Central Sulawesi, Indonesia that overlap across altitudinal ranges Note: asl = above sea level. 
Table 1. Macro fungus species found in Lore Rindu National Park, Central Sulawesi, Indonesia (WD: Wood debris, W: Wood, DL: Decomposing Litter, S: Soil, D: Dung)

\begin{tabular}{|c|c|c|c|}
\hline Species & Family & Division & Substrate \\
\hline Agaricus sp.1 & Agaricaceae & Basidiomycota & DL, $S$ \\
\hline Agaricus sp. 2 & Agaricaceae & Basidiomycota & $\mathrm{DL}, \mathrm{S}$ \\
\hline Agaricus sp.3 & Agaricaceae & Basidiomycota & DL, $S$ \\
\hline Agaricus sp.4 & Agaricaceae & Basidiomycota & DL, $S$ \\
\hline Agaricus lactifluus (L.) Pers & Agaricaceae & Basidiomycota & $\mathrm{DL}, \mathrm{S}$ \\
\hline Amanita sp.1 & Amanitaceae & Basidiomycota & $\mathrm{DL}, \mathrm{S}$ \\
\hline Amanita sp. 2 & Amanitaceae & Basidiomycota & DL, $S$ \\
\hline Amanita craseoderma Bas & Amanitaceae & Basidiomycota & $\mathrm{DL}, \mathrm{S}$ \\
\hline Amanita pantherina (DC.) Krombh. & Amanitaceae & Basidiomycota & $\mathrm{DL}, \mathrm{S}$ \\
\hline Amanita phalloides (Vaill. ex Fr.) Link & Amanitaceae & Basidiomycota & $\mathrm{DL}, \mathrm{S}$ \\
\hline Amauroderma rugosum (Blume \& T.Nees) Torrend & Ganodermataceae & Basidiomycota & $\mathrm{DL}, \mathrm{S}$ \\
\hline Agrocybe sp.1 & Strophariaceae & Basidiomycota & DL \\
\hline Agrocybe sp.2 & Strophariaceae & Basidiomycota & DL \\
\hline Agrocybe sp. 3 & Strophariaceae & Basidiomycota & DL \\
\hline Agrocybe parasitica & Strophariaceae & Basidiomycota & $\mathrm{W}$ \\
\hline Agrocybe pediades (Fr.) Fayod & Strophariaceae & Basidiomycota & DL, D \\
\hline Agrocybe retigera (Speg.) Singer & Strophariaceae & Basidiomycota & DL \\
\hline Auricularia sp.1 & Auriculariaceae & Basidiomycota & $\mathrm{WD}, \mathrm{W}$ \\
\hline Auricularia sp. 2 & Auriculariaceae & Basidiomycota & $\mathrm{WD}, \mathrm{W}$ \\
\hline Auricularia sp. 3 & Auriculariaceae & Basidiomycota & $\mathrm{WD}, \mathrm{W}$ \\
\hline Auricularia asiatica & Auriculariaceae & Basidiomycota & $\mathrm{WD}, \mathrm{W}$ \\
\hline Auricularia auricula-judae (Bull.) Quél. & Auriculariaceae & Basidiomycota & $\mathrm{WD}, \mathrm{W}$ \\
\hline Auricularia delicata (Mont.) Henn. & Auriculariaceae & Basidiomycota & $\mathrm{WD}, \mathrm{W}$ \\
\hline Auricularia polytricha & Auriculariaceae & Basidiomycota & WD, W \\
\hline Auricularia squamosa & Auriculariaceae & Basidiomycota & WD, W \\
\hline Bisporella sp. & Helotiaceae & Ascomycota & WD \\
\hline Bisporella citrina (Batsch) Korf \& S.E.Carp. & Helotiaceae & Ascomycota & WD \\
\hline Bisporella sulfurina & Helotiaceae & Ascomycota & WD \\
\hline Boletus sp.1 & Boletaceae & Basidiomycota & DL, $S$ \\
\hline Boletus sp.2 & Boletaceae & Basidiomycota & $\mathrm{DL}, \mathrm{S}$ \\
\hline Boletus sp. 3 & Boletaceae & Basidiomycota & $\mathrm{DL}, \mathrm{S}$ \\
\hline Boletus sp.4 & Boletaceae & Basidiomycota & $\mathrm{DL}, \mathrm{S}$ \\
\hline Clavulina cinereai (Bull.) J.Schröt. & Clavulinaceae & Basidiomycota & $\mathrm{S}$ \\
\hline Conocybe sp. & Bolbitiaceae & Basidiomycota & $\mathrm{DL}, \mathrm{S}$ \\
\hline Cookeina sp. 1 & Sarcoscyphaceae & Ascomycota & WD \\
\hline Cookeina sp.2 & Sarcoscyphaceae & Ascomycota & WD \\
\hline Cookeina sulcipes (Berk.) Kuntze & Sarcoscyphaceae & Ascomycota & WD \\
\hline Cookeina tricholoma (Mont.) Kuntze & Sarcoscyphaceae & Ascomycota & WD \\
\hline Copelandia sp. & Bolbitiaceaea & Basidiomycota & $\mathrm{DL}, \mathrm{S}$ \\
\hline Coprinellus disseminatus (Pers.) J.E.Lange & Psathyrellaceae & Basidiomycota & WD \\
\hline Coprinus sp. & Agaricaceae & Basidiomycota & $\mathrm{DL}, \mathrm{S}$ \\
\hline Coprinus plicatilis (Curtis) Redhead et al. & Agaricaceae & Basidiomycota & $\mathrm{DL}, \mathrm{S}$ \\
\hline Crepidotus sp. & Inocybaceae & Basidiomycota & WD \\
\hline Crinipellis sp.1 & Marasmiaceae & Basidiomycota & WD \\
\hline Crinipellis sp.2 & Marasmiaceae & Basidiomycota & WD \\
\hline Cyathus sp. & Agaricaceae & Basidiomycota & WD, DL \\
\hline Cyathus striatus (Huds.) Willd. & Agaricaceae & Basidiomycota & $\mathrm{WD}, \mathrm{DL}$ \\
\hline Daedaleopsis sp.1 & Polyporaceae & Basidiomycota & $\mathrm{W}$ \\
\hline Daedaleopsis sp.1 & Polyporaceae & Basidiomycota & $\mathrm{W}$ \\
\hline Dendrothele sp. & Nicaea & Basidiomycota & $\mathrm{WD}, \mathrm{W}$ \\
\hline Dictyophora indusiata Vent. & Phallaceae & Basidiomycota & $\mathrm{DL}, \mathrm{S}$ \\
\hline Entoloma sp. & Entolomataceae & Basidiomycota & $\mathrm{DL}, \mathrm{S}$ \\
\hline Entoloma nitidum & Entolomataceae & Basidiomycota & DL, $S$ \\
\hline Entoloma pachypus & Entolomataceae & Basidiomycota & $\mathrm{DL}, \mathrm{S}$ \\
\hline Exidia recisa (Ditmar) Fr. & Auriculariaceae & Basidiomycota & WD \\
\hline Galerina velutipes & Hymenogastraceaea & Basidiomycota & $\mathrm{WD}, \mathrm{W}$ \\
\hline Ganoderma sp.1 & Ganodermataceae & Basidiomycota & $\mathrm{WD}, \mathrm{W}$ \\
\hline Ganoderma sp.2 & Ganodermataceae & Basidiomycota & $\mathrm{WD}, \mathrm{W}$ \\
\hline Ganoderma sp.3 & Ganodermataceae & Basidiomycota & WD, W \\
\hline Ganoderma applanatum (Pers.) Pat. & Ganodermataceae & Basidiomycota & $\mathrm{WD}, \mathrm{W}$ \\
\hline Ganoderma lucidum Karst & Ganodermataceae & Basidiomycota & $\mathrm{WD}, \mathrm{W}$ \\
\hline Ganoderma nitidum & Ganodermataceae & Basidiomycota & WD, W \\
\hline
\end{tabular}




\begin{tabular}{|c|c|c|c|}
\hline Geastrum sp. & Geastraceaea & Basidiomycota & DL, WD \\
\hline Geastrum mirabile Mont. & Geastraceaea & Basidiomycota & DL, WD \\
\hline Geastrum rufescens Pers. & Geastraceaea & Basidiomycota & DL, WD \\
\hline Gerronema albidum & Marasmiaceae & Basidiomycota & WD \\
\hline Gomphidius sp.1 & Gomphidiaceae & Basidiomycota & DL. $S$ \\
\hline Gomphidius sp.2 & Gomphidiaceae & Basidiomycota & DL, $S$ \\
\hline Gymnopilus sp. & Hymenogastraceae & Basidiomycota & $\mathrm{W}$ \\
\hline Hexagonia apiaria (Pers.) Fr. & Polyporaceae & Basidiomycota & WD, W \\
\hline Hydropus marginellus & Marasmiaceae & Basidiomycota & DL, WD \\
\hline Hygrocybe sp.1 & Hygrophoraceae & Basidiomycota & $\mathrm{DL}, \mathrm{S}$ \\
\hline Hygrocybe sp.2 & Hygrophoraceae & Basidiomycota & DL, $\mathrm{S}$ \\
\hline Hygrocybe sp. 3 & Hygrophoraceae & Basidiomycota & DL, $S$ \\
\hline Hygrocybe sp.4 & Hygrophoraceae & Basidiomycota & DL, $S$ \\
\hline Hygrocybe sp.5 & Hygrophoraceae & Basidiomycota & DL, $S$ \\
\hline Hygrocybe sp.6 & Hygrophoraceae & Basidiomycota & DL, $S$ \\
\hline Hygrocybe sp.7 & Hygrophoraceae & Basidiomycota & DL, $S$ \\
\hline Hygrocybe coccineocrenata (P.D. Orton) M.M. Moser & Hygrophoraceae & Basidiomycota & DL, $\mathrm{S}$ \\
\hline Hygrocybe conicoides (P.D. Orton) P.D. Orton \& Watling & Hygrophoraceae & Basidiomycota & DL, $S$ \\
\hline Hygrocybe constrictospora Arnolds & Hygrophoraceae & Basidiomycota & DL, $S$ \\
\hline Hygrocybe nitida (Berk. \& M.A.Curtis) Murrill & Hygrophoraceae & Basidiomycota & DL, $S$ \\
\hline Hygrocybe psittacina (Schaeff.) P. Kumm. & Hygrophoraceae & Basidiomycota & $\mathrm{DL}, \mathrm{S}$ \\
\hline Hygrophorus sp. & Hygrophoraceae & Basidiomycota & $\mathrm{DL}, \mathrm{S}$ \\
\hline Hymenochaete tabacina (Sowerby) Lév. & Hymenochaeteceae & Basidiomycota & WD, W \\
\hline Lactifluus sp. & Russulaceae & Basidiomycota & $\mathrm{DL}, \mathrm{S}$ \\
\hline Lactifluus acris (Bolton) Gray & Russulaceae & Basidiomycota & $\mathrm{DL}, \mathrm{S}$ \\
\hline Lactifluus aspideus (Fr.) Fr. & Russulaceae & Basidiomycota & $\mathrm{DL}, \mathrm{S}$ \\
\hline Lactifluus aquifluus (Peck) Kuntze & Russulaceae & Basidiomycota & DL, $S$ \\
\hline Lactifluus bicolor (Massee) & Russulaceae & Basidiomycota & DL, $S$ \\
\hline Lactifluus corrugis (Peck) Kuntze & Russulaceae & Basidiomycota & $\mathrm{DL}, \mathrm{S}$ \\
\hline Lactifluus curtus & Russulaceae & Basidiomycota & $\mathrm{DL}, \mathrm{S}$ \\
\hline Laetiporus sp. & Fomitopsidaceae & Basidiomycota & WD, W \\
\hline Laetiporus discolor (Klotzsch) Corner & Fomitopsidaceae & Basidiomycota & WD, W \\
\hline Lentinus sp. & Polyporaceae & Basidiomycota & $\mathrm{DL}, \mathrm{S}$ \\
\hline Lentinus ciliatus Lév. & Polyporaceae & Basidiomycota & $\mathrm{DL}, \mathrm{S}$ \\
\hline Lentinus sajor-caju (Fr.) Fr. & Polyporaceae & Basidiomycota & DL, $S$ \\
\hline Leucocoprinus fragilissimus (Berk. \& M.A.Curtis) Pat. & Agaricaceae & Basidiomycota & $\mathrm{DL}$ \\
\hline Marasmius sp.1 & Marasmiaceae & Basidiomycota & DL, WD \\
\hline Marasmius sp.2 & Marasmiaceae & Basidiomycota & DL, WD \\
\hline Marasmius sp. 3 & Marasmiaceae & Basidiomycota & DL, WD \\
\hline Marasmius sp.4 & Marasmiaceae & Basidiomycota & DL, WD \\
\hline Marasmius androsaceus & Marasmiaceae & Basidiomycota & DL, WD \\
\hline Marasmius botryoides & Marasmiaceae & Basidiomycota & DL, WD \\
\hline Marasmius delectans & Marasmiaceae & Basidiomycota & DL, WD \\
\hline Marasmius maximus Hongo & Marasmiaceae & Basidiomycota & DL, WD \\
\hline Marasmius papyraceus & Marasmiaceae & Basidiomycota & DL, WD \\
\hline Marasmius pellucidus & Marasmiaceae & Basidiomycota & DL, WD \\
\hline Marasmius purpureostriatus & Marasmiaceae & Basidiomycota & DL, WD \\
\hline Marasmius rotula (Scop.) Fr. & Marasmiaceae & Basidiomycota & DL, WD \\
\hline Microporus xanthopus (Fr.) Kuntze & Polyporaceae & Basidiomycota & DL, WD \\
\hline Mycena sp.1 & Mycenaceae & Basidiomycota & DL, WD \\
\hline Mycena sp.2 & Mycenaceae & Basidiomycota & DL, WD \\
\hline Mycena stylobates (Pers.) P.Kumm. & Mycenaceae & Basidiomycota & DL, WD \\
\hline Phallus sp. 1 & Phallaceae & Basidiomycota & $\mathrm{DL}, \mathrm{WD}, \mathrm{S}$ \\
\hline Phallus sp.2 & Phallaceae & Basidiomycota & $\mathrm{DL}, \mathrm{WD}, \mathrm{S}$ \\
\hline Phallus sp.3 & Phallaceae & Basidiomycota & $\mathrm{DL}, \mathrm{WD}, \mathrm{S}$ \\
\hline Phallus subtilis (Möller) Lloyd & Phallaceae & Basidiomycota & DL, WD, S \\
\hline Phalus vulgaris Micheli & Phallaceae & Basidiomycota & DL, WD, S \\
\hline Phillipsia domingensis Berk. & Sarcoscyphaceae & Ascomycota & WD \\
\hline Phillipsia subpurpurea Berk. \& Broome & Sarcoscyphaceae & Ascomycota & WD \\
\hline Picnoporus sp.1 & Polyporaceae & Basidiomycota & $\mathrm{WD}, \mathrm{W}$ \\
\hline Picnoporus sp.2 & Polyporaceae & Basidiomycota & $\mathrm{WD}, \mathrm{W}$ \\
\hline Pycnoporus cinnabarinus (Jacq.) P.Karst. & Polyporaceae & Basidiomycota & $\mathrm{WD}, \mathrm{W}$ \\
\hline Pycnoporus sanguineus (L.) Murrill & Polyporaceae & Basidiomycota & $\mathrm{WD}, \mathrm{W}$ \\
\hline Pleurotus sp.1 & Pleurotaceae & Basidiomycota & WD \\
\hline Pleurotus sp.2 & Pleurotaceae & Basidiomycota & WD \\
\hline Pleurotus niger & Pleurotaceae & Basidiomycota & WD \\
\hline Pleurotus ornatus (Fr.) Sacc. & Tricholomataceae & Basidiomycota & WD \\
\hline
\end{tabular}




\begin{tabular}{|c|c|c|c|}
\hline Pleurotus ostreatus (Jacq. ex Fr.) P.Kumm. & Pleurotaceae & Basidiomycota & WD \\
\hline Polyporus sp.1 & Polyporaceae & Basidiomycota & WD, W \\
\hline Polyporus sp.2 & Polyporaceae & Basidiomycota & $\mathrm{WD}, \mathrm{W}$ \\
\hline Polyporus abietinus (Dicks.) Fr. & Polyporaceae & Basidiomycota & WD, W \\
\hline Polyporus abruptus & Polyporaceae & Basidiomycota & $\mathrm{WD}, \mathrm{W}$ \\
\hline Polyporus acutus & Polyporaceae & Basidiomycota & WD, W \\
\hline Polyporus angulatus Schumach. & Polyporaceae & Basidiomycota & $\mathrm{WD}, \mathrm{W}$ \\
\hline Polyporus squamosus & Polyporaceae & Basidiomycota & WD, W \\
\hline Poromycena sp. & Mycenaceae & Basidiomycota & WD \\
\hline Psilocybe coprophila (Bull.) P.Kumm. & Hymenogastraceae & Basidiomycota & $\mathrm{D}$ \\
\hline Ramaria sp.1 & Gomphaceae & Basidiomycota & DL, WD \\
\hline Ramaria sp. 2 & Gomphaceae & Basidiomycota & DL, WD \\
\hline Russula sp.1 & Russulaceae & Basidiomycota & $\mathrm{DL}, \mathrm{S}$ \\
\hline Russula sp.2 & Russulaceae & Basidiomycota & DL,S \\
\hline Russula sp.3 & Russulaceae & Basidiomycota & DL,S \\
\hline Russula acris Steinhaus & Russulaceae & Basidiomycota & $\mathrm{DL}, \mathrm{S}$ \\
\hline Russula admirabilis Beardslee \& Burl. & Russulaceae & Basidiomycota & $\mathrm{DL}, \mathrm{S}$ \\
\hline Russula aeruginosa & Russulaceae & Basidiomycota & $\mathrm{DL}, \mathrm{S}$ \\
\hline Russula albonigra (Krombh.) Fr. & Russulaceae & Basidiomycota & $\mathrm{DL}, \mathrm{S}$ \\
\hline Russula alutacea (Pers.) Fr. & Russulaceae & Basidiomycota & $\mathrm{DL}, \mathrm{S}$ \\
\hline Russula amoenicolor Romagn. & Russulaceae & Basidiomycota & DL,S \\
\hline Russula annulata & Russulaceae & Basidiomycota & $\mathrm{DL}, \mathrm{S}$ \\
\hline Russula campinensis & Russulaceae & Basidiomycota & $\mathrm{DL}, \mathrm{S}$ \\
\hline Schizophyllum sp.1 & Schizophyllaceae & Basidiomycota & WD \\
\hline Schizophyllum sp.2 & Schizophyllaceae & Basidiomycota & WD \\
\hline Schizophyllum commune Fries & Schizophyllaceae & Basidiomycota & WD \\
\hline Stereum ostrea (Blume \& T. Nees ex Fr. & Stereaceae & Basidiomycota & WD,W \\
\hline Termitomyces sp. & Lyophyllaceae & Basidiomycota & $\mathrm{DL}, \mathrm{S}$ \\
\hline Termitomyces clypeatus R.Heim & Lyophyllaceae & Basidiomycota & $\mathrm{DL}, \mathrm{S}$ \\
\hline Termitomyces eurrhizus (Berk.) R. Heim & Lyophyllaceae & Basidiomycota & $\mathrm{DL}, \mathrm{S}$ \\
\hline Trametes sp. 1 & Polyporaceae & Basidiomycota & $\mathrm{WD}, \mathrm{W}$ \\
\hline Trametes sp. 2 & Polyporaceae & Basidiomycota & $\mathrm{WD}, \mathrm{W}$ \\
\hline Trametes sp. 3 & Polyporaceae & Basidiomycota & WD,W \\
\hline Trametes elegans (Spreng.:Fr.) Fr. & Polyporaceae & Basidiomycota & WD,W \\
\hline Trametes gibbosa (Pers.) Fr. & Polyporaceae & Basidiomycota & WD,W \\
\hline Trametes versicolor (L.) Lloyd & Polyporaceae & Basidiomycota & $\mathrm{WD}, \mathrm{W}$ \\
\hline Tremella mesenterica Retz. & Tremellaceae & Basidiomycota & WD \\
\hline Trogia infundibuliformis & Marasmiaceae & Basidiomycota & WD \\
\hline Vanromburghia silvetris Holterm. & Marasmiaceae & Basidiomycota & WD \\
\hline Xylaria sp.1 & Xylariaceae & Ascomycota & WD \\
\hline Xylaria sp. 2 & Xylariaceae & Ascomycota & WD \\
\hline Xylaria sp. 3 & Xylariaceae & Ascomycota & WD \\
\hline Xylaria nigripes & Xylariaceae & Ascomycota & WD \\
\hline
\end{tabular}

\section{Ethnomycology}

The results of interview and discussion with the residents in the buffer zone of the national park showed that they were familiar with macrofungus that they used for food and medicinal purposes. They harvested fungal bodies that were found in the parking area or their yard. These were then sold to traditional markets to add cash incomes. Residents called small-sized fungi as "Tangidi" or "Tanggojo," two names that appear popular among the ethnic Kaili-Da'a tribe or in the Lore tribe in the buffer zones of the national park. The macrofungus Schyzophyllum commune was known by the residents of Kaili as a food source because it has a good substrate to grow on, and is easier to collect and find, especially in the rainy season. These types of fungi have more diverse substrates to grow on, and frequently grow in rotten-wood of fallen trees, such as candlenut trunk (Aleurites moluccana), Pine (Pinus merkusii), Gamal (Gliricidia sepium), Bayan tree (Ficus sp), Mango (Mangifera spp), Java wood (Lannea coromandelica), Cassava (Manihot esculenta), River tamarind/Lamtoro (Leucaena leucocephala), Peacock plum/Sengon (Paraserianthes falcataria L. Nielsen), Milkwood-pine/Pulai (Alstonia $\mathrm{sp}$ ), Siamese cassia/Johar (Senna siamea), Areng palm (Arenga pinnata), Teak (Tectona grandis L), Coconut (Cocos nucifera), Gebang palm (Corypha sp), Macaranga sp., etc. The macro fungus Lentinus sajor-caju was also found in some rotten-wood from the Burflower-tree/Jabon (Anthocephalus cadamba), Mango (Mangifera spp), Bayan tree (Ficus sp) and others. Phallus sp., which appears in the rainy season, is often believed by residents to be spiritual/ghost-related, representing a "male-ghost" because the trunk of the fungus is similar in shape to that of a human penis, and they strongly produce a bad-odor. Residents commonly called it "Lasu Mboa" (Lasu= penis, Mboa $=$ ghost). The naming of macrofungus by residents is often influenced by the growth place of the fungi. Fungi found in rotten mango trunks, for example, are given the name "Tanggidi/Tanggojo Taipa" (Tanggidi/Tanggojo= fungus, Taipa $=$ mango). 


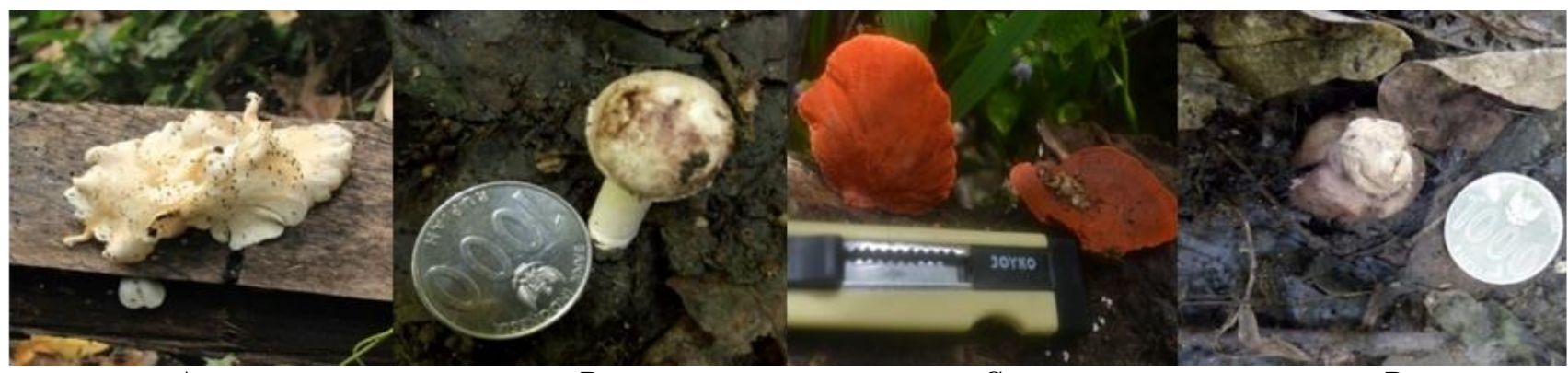

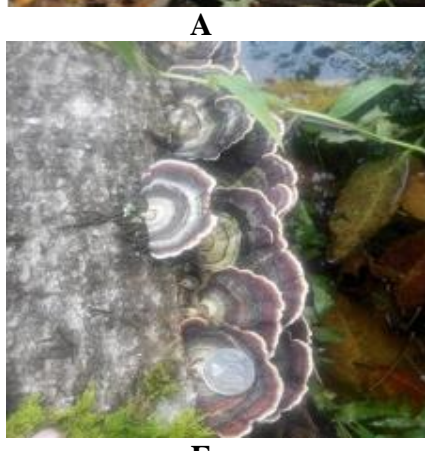

$\mathbf{E}$

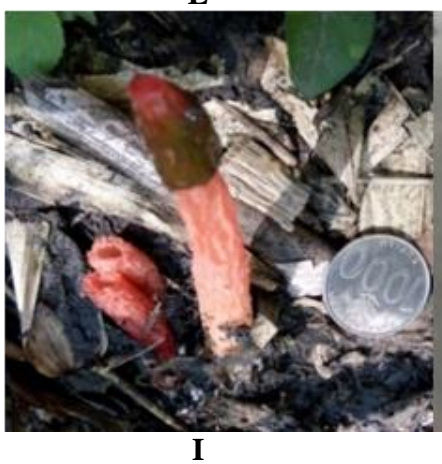

C

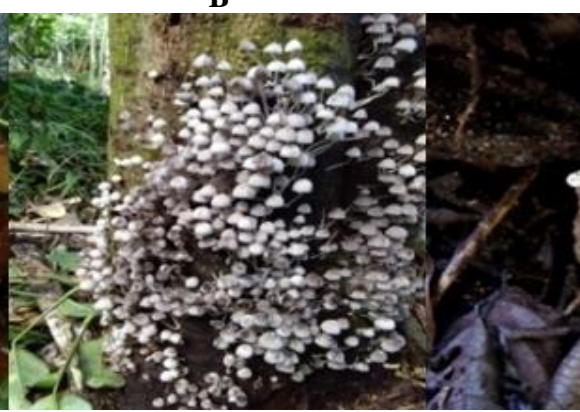

F

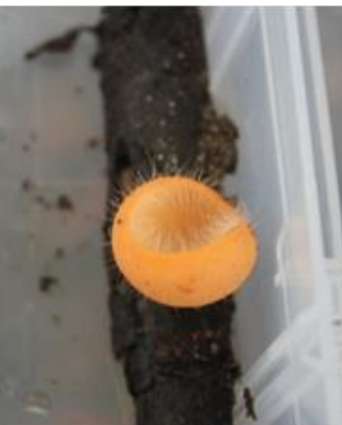

J

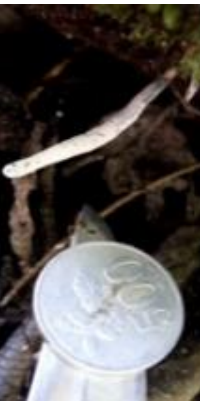

G

D

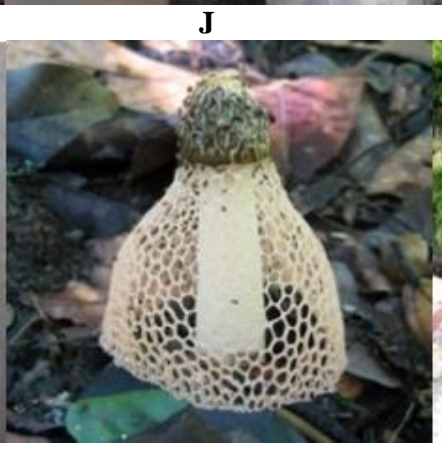

N

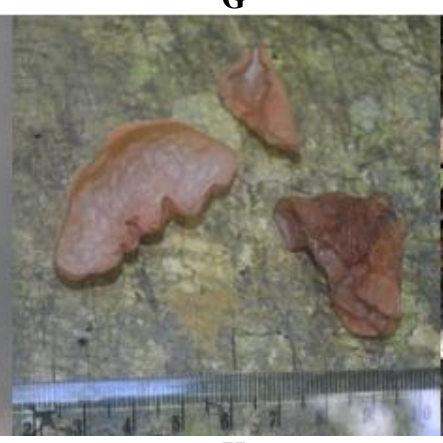

$\mathbf{K}$
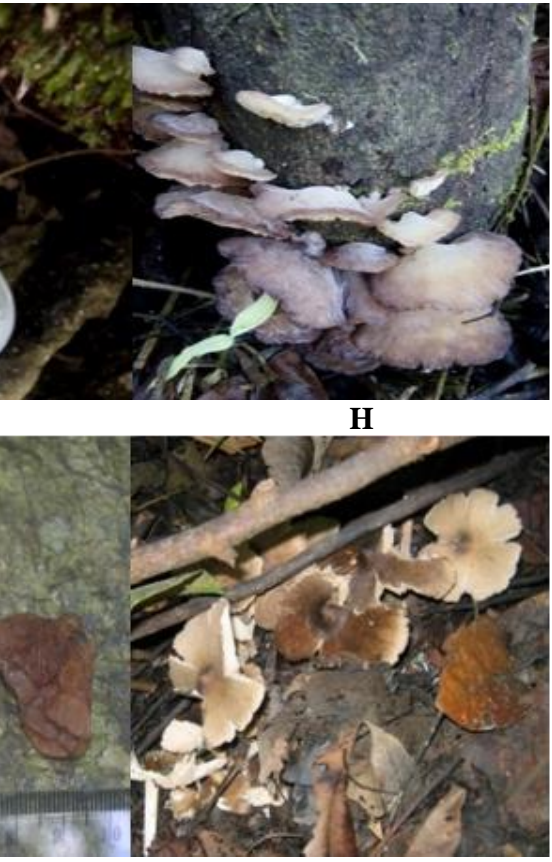

L

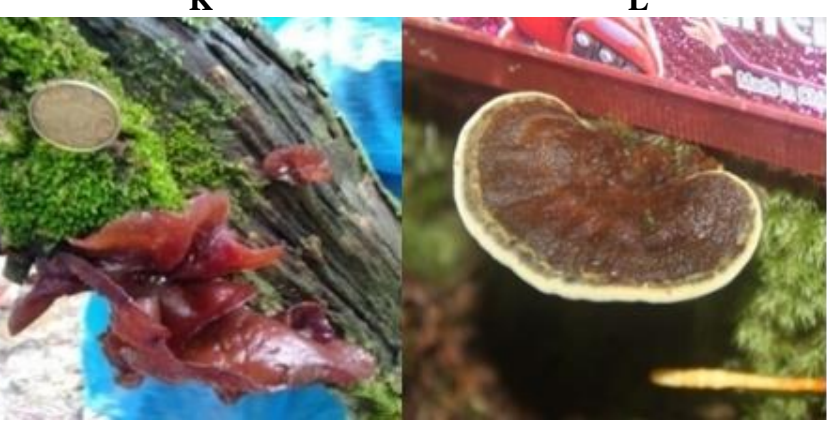

$\mathbf{O}$

$\mathbf{P}$
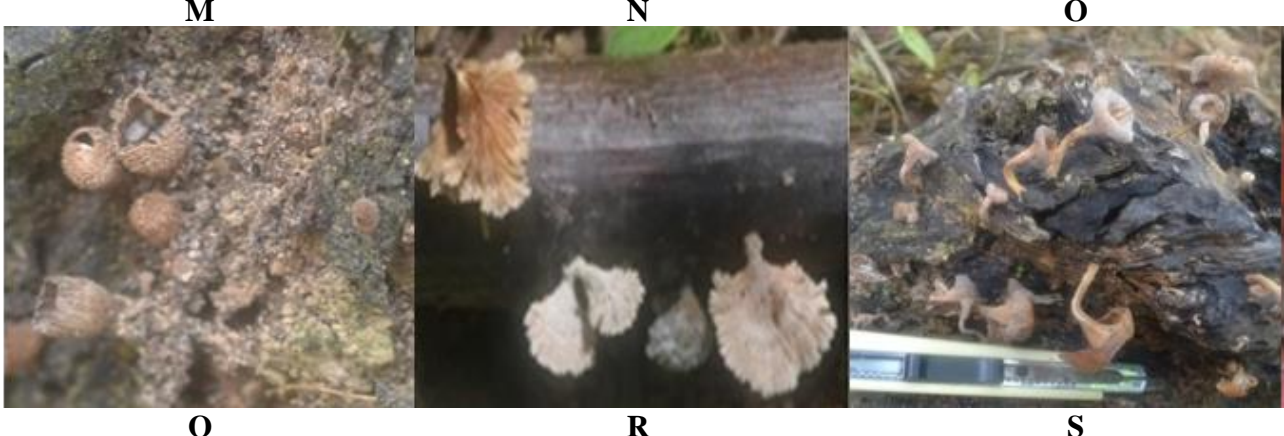

$\mathbf{S}$

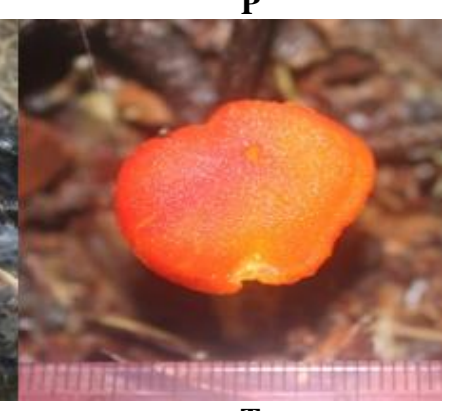

T

Figure 5. A. Pleurotus ostreatus (Jacq. ex Fr.) P.Kumm., B. Lycoperdon sp., C. Picnoporus sp., D. Geastrum sp., E. Trametes versicolor (L.) Lloyd, F. Marasmius sp., G. Xylaria sp., H. Pleurotus sp.1., I. Phallus sp., J. Cookeina tricholoma (Mont.) Kuntze., K. Auricularia auricula-judae (Bull.) Quél., L. Termytomyces sp., M. Ramaria sp., N. Dictyophora indusiata Vent., O. Auricularia sp.1, P. Ganoderma lucidum Kars, Q. Cyathus sp., R. Schizophyllum commune Fries., S. Lentinus sajor-caju (Fr.) Fr., T. Hygrocybe sp. 
The common name of the large-sized macro fungus is "Tambata," and has been given to members of the genera Pleurotus sp, Lentinus sarjo-caju, and Auricularia sp. These results show the same naming trends as found by Teke et al. (2018) in the Northeast Region of Cameroon, where the residents gave names to fungi based on their growth substrates.

The major reason why residents use these fungi was that the edible part of the fungus is highly palatable. They also believed that once they consumed the fungus, their digestive system is smoother than usual, and they simultaneously fulfill their nutrient needs. However, those statements still need further experimental analysis. Still, from the interviews, residents were also familiar with some toxic fungus types. According to the residents, the features of toxic fungus are a brightly colored trunk that is not predated by insects (such as ants, termites, grasshopper, and beetles) or rodents (such as mouse and squirrels). In contrast, if the fruit body of the fungus is a white color, or tends to dark, and is visited by sticks insects, or other animals, they believed that the fungus is safe to consume. Similar indicators were reported by Teke et al. (2018) who describe that the "Killum-Ijim," in Northwest region of Cameroon, have different methods to determine whether the fungus could be eaten or not. Local knowledge that leads residents to distinguish the type of fungus is usually passed down between generations.

We found nine macrofungus species that are often used as source of food and medicine by the residents, such as Schizophyllum commune, Termytomyces sp, Auricularia auricular-judae, Auricularia sp, Pleurotus ostreatus, Ganoderma lucidum, Xylaria sp, Lentinus sajor-caju, and Agaricus sp. A similar exploration was done by Khastini et al. (2018), who found six species of macrofungus that were used as medicines by the Baduy tribe in Banten Province, Indonesia. Also, Rosemary-Kinge et al. (2017) found eight species of fungus that were also used as medicine and food sources by the residents of the Awing Wildlife Sanctuary in Cameroon. Another report found that 14 species of macrofungus were used as medicinal and food sources in Central Luzon, Philippines (De Leon et al. 2012), while 36 species were also used by the residents in Nueva Ecija, Philippines (De Leon et al. 2016). Traditional medicine plays an important role in the lives of the residents of the Kaili Tribe. They often use the macrofungus as a medicine source in first aid kits, and if the problem is still not solved, they prefer to go to a shaman (in Kaili language $=$ Sando), who can usually be found in each village. Pictures of the documented macrofungus are shown in Figure 5.

In conclusion, we found 172 species (including unidentified species) of macrofungus in the Lore Lindu National Park area. Twenty-four species of the family Polyporaceae were found, making it the most abundant family. Marasmius spp. and Hygrocybe spp. were the most abundant genera. Species diversity of macrofungus increased alongside the increase in altitude of the sampling locations. Furthermore, we found nine species of macrofungus that were intensively used as food and medicinal sources by the local residents. The results of this study can serve as basic data of macrofungus diversity in the area to complement the existing biodiversity data. The information can be used as reference for the management of Lore Lindu National Park in the future. Nonetheless, future research on the topics of domestication of edible and medicinal fungus found in this study, especially regarding commercialization, are still required.

\section{ACKNOWLEDGEMENTS}

The author thanks Higher Education Directory, Ministry of Higher Education, Research and Technology, the Republic of Indonesia for the given Penelitian Terapan Unggulan Perguruan Tinggi (PTUPT) Research Grant No.699.h/UN28.2/PL/2017, 27 $7^{\text {th }}$ April 2017. The author also thanks Balai Besar Taman Nasional Lore Lindu (Lore Lindu National Park) for the permission to conduct the research and supporting facilities, and to Faculty of Forestry students; Andi, Mukrim, Imran, Risman, Rahmadiansyah, Firka Baido, Rizal Kurniawan, which support authors in the field collection and in the laboratory. The authors declare that there is no conflict of interest in this research.

\section{REFERENCES}

Andrew EE, Rosemary-Kinge T, Tabi EM, Thiobal N, Mih AM. 2013. Diversity and distribution of macrofungi (mushrooms) in the Mount Cameroon Region. J Ecol Nat Environ 5 (10): 318-334. DOI: 10.5897/JENE2013.0397.

Angelini P, Bistocchi G, Arcangeli A, Bricchi E, Venanzoni R. 2015. Diversity and ecological distribution of macrofungi in a site of community importance of Umbria (Central Italy). Open Ecol J 8: 1-8. DOI: $10.2174 / 1874213001508010001$.

Arko PF, Marzuki BM, Kusmoro J. 2017. The inventory of edible mushrooms in Kamojang Nature Reserve and Nature Park, West Java, Indonesia. Biodiversitas 18 (2): 530-540. DOI: 10.13057/biodiv/d180213.

Baptista P, Martins A, Tavares RM, Lino-Neto T. 2010. Diversity and fruiting pattern of macrofungi associated with chestnut (Castanea sativa) in the Trás-os-Montes region (Northeast Portugal). Fungal Ecol 3 (1): 9-19. DOI: 10.1016/j.funeco.2009.06.002.

Bates ST. 2006. A preliminary checklist of Arizona macrofungi. CANOTIA 2 (2): 47-78.

Bergemann SE, Largent DL. 2000. The site-specific variables that correlate with the distribution of the Pacific Golden Chanterelle, Cantharellus formosus. Forest Ecol Manag 130 (1): 99-107. DOI: 10.1016/S0378-1127(99)00177-2.

Boa E. 2004. Wild edible fungi: A global overview of their use and importance to people. Food and Agriculture Organization of the United Nations, Rome, Italy. http://www.fao.org/3/a-y5489e.pdf.

Chang YS, Lee SS. 2004. Utilisation of macrofungi species in Malaysia. Fungal Divers 15: 15-22.

Conn C, Dighton J. 2000. Litter quality influences on decomposition, ectomycorrhizal community structure and mycorrhizal root surface acid phosphatase activity. Soil Biol Biochem 32 (4): 489-496. DOI: 10.1016/S0038-0717(99)00178-9.

Corlett R, Primack R. 2006. Tropical Rain Forests: An Ecological and Biogeographical Comparison. Wiley-Blackwell, Hoboken, NJ.

De Leon AM, Kalaw SP, Dulay RM, Undan JR, Alfonzo DO, Undan JQ, Reyes RG. 2016. Ethnomycological survey of the Kalanguya indigenous community in Caranglan, Nueva Ecija, Philippines. Curr Res Environ Appl Mycol 6 (2): 61-66. DOI: 10.5943/cream/6/2/1.

De Leon AM, Reyes RG, dela Cruz TEE. 2012. An ethnomycological survey of macrofungi utilized by Aeta communities in Central Luzon, $\begin{array}{llll}\text { Philippines. } & \text { Mycosphere } 3 & \text { (2): 251-259. DOI: }\end{array}$ 10.5943/mycosphere/3/2/9. 
Demirbas A. 2000. Accumulation of heavy metals in some edible mushrooms from Turkey. Food Chem 68 (4): 415-419. DOI: 10.1016/S0308-8146(99)00210-1.

Desjardin DE, Flegel TW, Boonpratuang T. 2004. Basidiomycetes. In: Jones EBG, Tanticharoen M, Hyde KD (eds) Thai fungal diversity BIOTEC. Thailand.

Desjardin DE, Retnowati A, Horak E. 2000. Agaricales of Indonesia. 2. A Preliminary monograph of Marasmius from Java and Bali. Sydowia 52 (2): 92-194. [Indonesian]

Douanla-Meli C, Ryvarden L, Langer E. 2007. Studies of tropical African pore fungi (Basidiomycota, Aphyllophorales): Three new species from Cameroon. Nova Hedwigia 84(3-4): 409-420. DOI: 10.1127/0029-5035/2007/0084-0409.

Duarte S, Pascoal C, Cassio F, Bärlocher F. 2006. Aquatic hyphomycete diversity and identity affect leaf litter decomposition in microcosms. Oecologia 147 (4): 658-666.

Enas AE, Sababelkhier MKI, Malaz MM. 2016. Nutritional composition and minerals content of five species of wild edible mushroom, brought from UAE: Mushroom considered as protein source. Int J Curr Adv Res 4 (2): 1108-1112.

Engola APO, Eilu G, Kabasa JD, Kisovi L, Munishi PKT, Olila D. 2007. Ecology of edible indigenous mushrooms of the Lake Victoria Basin (Uganda). Res J Biol Sci 2 (1): 62-68.

Ferris R, Peace AJ, Newton AC. 2000. Macrofungal communities of lowland Scots pine (Pinus sylvestris L.) and Norway spruce (Picea abies (L.) Karsten.) plantations in England: Relationships with site factors and stand structure. For Ecol Manag 131 (1-3): 255-267. DOI: 10.1016/S0378-1127(99)00218-2.

Foo SF, Saikim FH, Kulip J, Seelan JSS. 2018. Distribution and ethnomycological knowledge of wild edible mushrooms in Sabah (Northern Borneo), Malaysia. J Trop Biol Conserv 15: 203-222.

Haryati JR, Azrianingsih DR. 2012. Ethno-edible mushroom of Pleurotus sp, Clytocybe nebularis and Auricularia auricular in Ranupani Village, East Java. J Trop Life Sci 2 (2): 40-43.

Hawksworth D, Lücking R. 2017. Fungal diversity revisited: 2.2 to 3.8 million species. Microbiol Spectr 5 (4): 79-95. DOI 10.1128/microbiolspec.FUNK-0052-2016.

Hemmes DE, Desjardin DE. 2002. Mushrooms of Hawai'i: An Identification Guide. Ten Speed Press, Berkeley, CA

Herawati E, Arung ET, Amirta R. 2016. Domestication and nutrient analysis of Schizophyllum commune, alternative natural food sources in East Kalimantan. Agric Agric Sci Procedia 9: 291-296. DOI: 10.1016/j.aaspro.2016.02.125.

Hussein JM, Tibuhwa DD, Mshandete MM, Kivaisi AK. 2016. Successful domestication of Lentinus sajor-caju from an indigenous forest in Tanzania. J Appl Biosci 108: 10500-10506.

Index Fungorum 2017. http://www.indexFungorum.org/names.

Kalac P, Svoboda L, Havlickova B. 2004. Content of cadmium and mercury in edible mushrooms. J Appl Biomed 2 (1): 15-20. DOI: $10.32725 / \mathrm{jab} .2004 .002$

Kang Y, Łuczaj L, Kang J, Zhang S. 2013. Wild food plants and wild edible fungi in two valleys of the Qinling Mountains (Shaanxi, Central China). J Ethnobiol Ethnomed 9: 26. DOI: 10.1186\%2F17464269-9-26.

Khastini RO, Wahyuni I, Saraswati I. 2018. Ethnomycology of bracket fungi in Baduy Tribe Indonesia. Biosaintifika 10 (2): 423-431. DOI: 10.15294/biosaintifika.v10i2.14082.

Kim H, Song MJ. 2014. Analysis of traditional knowledge for wild edible mushrooms consumed by residents living in Jirisan National Park $\begin{array}{lllll}\text { (Korea). J Ethnopharmacol } 153 & \text { (1): 90-97. DOI }\end{array}$ 10.1016/j.jep.2013.12.041

Kutszegi G, Siller I, Dima B, Takács K, Merényi Z, Varga T, Ódor P. 2015. Drivers of macrofungal species composition in temperate forest, West Hungary: Functional groups compared. Fungal Ecol 17: 69-83. DOI: 10.1016/j.funeco.2015.05.009.

Lopez-Quintero CA, Straatsma G, Franco-Molano AE, Boekhout T. 2012. Macrofungal diversity in Colombian Amazon forests varies with regions and regimes of disturbance. Biodivers Conserv 21 (9): 22212243. DOI: $10.1007 / \mathrm{s} 10531-012-0294-2$

Luo X, Karunarathna SC, Luo YH, Xu K, Xu JC, Chamyuang S, Mortimer PE. 2016. Drivers of macrofungal composition and distribution in Yulong Snow Mountain, Southwest China. Mycosphere 7 (6): 727-740. DOI: 10.5943/mycosphere/7/6/3

Manzi P, Gambelli L, Marconi S, Vivanti V, Pizzoferrato L. 1999. Nutrients in edible mushroom: An interspecies comparative study Food Chem 65 (4): 477-482. DOI: 10.1016/S0308-8146(98)00212-X.
Marzuki BM, Rossiana N, Normanita. 2017. Diversity of macrofungi on wood in forest nature reserve of Bojonglarang Jayanti Cianjur, West Java. J Bacteriol Mycol 4 (1): 25-28. DOI: 10.15406/jbmoa.2017.4.00080

Muchlisin ZA, Azizah MNS. 2009. Diversity and distribution of freshwater fishes in Aceh water, Northern-Sumatra, Indonesia. Int J Zool Res 5 (2) 62-79. DOI: 10.3923/ijzr.2009.62.79.

Mueller GM, Schmit JP, Leacock PR, Buyck B, Cifuentes J, Desjardin $\mathrm{DE}$, Halling RE, Iturriaga T, Larsson KH, Lodge DJ, May TW, Minter D, Rajchenberg M, Redhead SA, Ryvarden L, Trappe JM, Watling R, Wu Q. 2007. Global diversity and distribution of macrofungi. Biodivers Conserv 16 (1): 37-48. DOI: 10.1007/s10531006-9108-8.

Mushroom Expert. 2017. http://mushroomexpert.com/.

Nema JK, Wossa S, Costillo E, Marasinghe B, Pooranalingam J, Barrow R. 2014. Ethno mycological survey and biological activities of mushroom extracts against Escherichia coli and Staphylococcus epidermis from Papua New Guinea. Biol Chem Res 2014: 97-103.

Okhuoya JA, Akpaja EO, Osemwegie OO, Oghenekaro AO, Ihayere CA. 2010. Nigerian mushrooms: Underutilized non-wood forest resources. J Appl Sci Environ Manag 14 (1): 43-54. DOI: 10.4314/jasem.v14i1.56488.

Packham JM, May TW, Brown MJ, Wardlaw TJ, Mills KA. 2002. Macrofungal diversity and community ecology in mature and regrowth wet eucalypt forest in Tasmania: A multivariate study. Austral Ecol 27 (2): 149-161. DOI: 10.1046/j.14429993.2002.01167.x.

Pitopang R. 2006. Structure and Composition of Six Land Use Types in the Lore Lindu National Park, Central Sulawesi. [Dissertation]. School of Graduates, Bogor Agriculture University, Bogor. [Indonesian]

Pushpa H, Purushothama KB. 2012. Biodiversity of mushrooms in and around Bangalore (Karnataka), India. Am Eurasian J Agric Environ Sci 12 (6): 750-759.

Retnowati A. 2007. Two wild edible Russula (Agaricales: Russulaceae) from East Kalimantan. Floribunda 3 (4): 109-112. DOI: 10.32556/floribunda.v3i3-4.2007.78

Rosemary-Kinge T, Apalah NA, Nji TM, Acha AN, Mih AM. 2017. Species richness and traditional knowledge of macrofungi (Mushrooms) in the Awing forest reserve and communities, Northwest Region, Cameroon. J Mycol 17: 2809239. DOI: $10.1155 / 2017 / 2809239$.

Rosemary-Kinge T, Tabi EM, Mih AM, Enow EA, Njouonkou L, Nji TM. 2011. Etnomycological studies of edible and medicinal mushrooms in the Mount Cameroon region (Cameroon, Africa). Int $\mathrm{J}$ Med Mushrooms 13 (3): 299-305. DOI: 10.1615/intjmedmushr.v13.i3.100

Sarbhoy AK, Varshney JL, Agarwal DK. 1996. Fungi of India 1982-1992. CBS Publishers and Distributors, New Delhi.

SK MenHut RI. No.593/Kpts-II/93, Tanggal 5 Oktober 1993

Stadler M, Laessøe T, Fournier J, Decock C, Schmieschek B, Tichy HV, Peršoh D. 2014. A polyphasic taxonomy of Daldinia (Xylariaceae) Stud Mycol 77 (1): 1-143. DOI: 10.3114/sim0016

Tan X, Mi F, Zhang Y, He X, Cao Y, Wang P, Xu J. 2015. Diversity, population genetics, and evolution of macrofungi associated with $\begin{array}{lllll}\text { animal. Mycology } 6 & \text { (2): 94-109. DOI: }\end{array}$ 10.1080/21501203.2015.1043968.

Teke NA, Rosemary-Kinge T, Bechem E, Nji TM, Ndam LM, Mih AM. 2018. Ethnomycological study in the Kilum-Ijim mountain forest, Northwest Region, Cameroon. J Ethnobiol Ethnomed 14: 25. DOI: $10.1186 \% 2$ Fs $13002-018-0225-8$.

TNC. 2001. Community Consultations Provide Key to Lore Lindu Success. The Nature Conservancy and Wahana Lingkungan Hidup, Jakarta, Indonesia. [Indonesian]

TNC/BTNLL. 2002. Lore Lindu National Park. Draft Management Plant 2002-2007. Directorate General of Forest Protection and Nature Conservation, Palu, Indonesia. [Indonesian]

Tran NH, Urase T, Kusakabe O. 2010. Biodegradation characteristics of pharmaceutical substances by whole fungal culture Trametes versicolor and its laccase. J Water Environ Tech 8 (2): 125-140. DOI: 10.2965/jwet.2010.125

Tran NH, Urase T, Ngo HH, Hu J, Ong SL. 2013. Insight into metabolic and cometabolic activities of autotrophic and heterotrophic microorganisms in the biodegradation of emerging trace organic contaminants. Bioresour Technol 146: 721-731. DOI: 10.1016/j.biortech.2013.07.083. 
Van Balgooy MMJ, Hovenkamp PH, Welzen PC. 1996. Phytogeography of the Pacific-Floristic and historical distribution pattern in plant. In: Keast A, Miller SE (eds) The Origin and Evolution of Pacific Island Biotas, New Guinea to Eastern Polynesia: Pattern and Processes. SPB Academic Publishing, Amsterdam.
Wallace AR. 1869. The Malay Archipelago. Harper and Brothers, New York.

Whitten AJ, Mustafa M, Henderson GS. 1987. The Ecology of Sulawesi. Gadjah Mada University Press, Yogyakarta, Indonesia. [Indonesian] 